\title{
Time required for disappearance of urate crystals from synovial fluid after successful hypouricaemic treatment relates to the duration of gout
}

\author{
Eliseo Pascual, Francisca Sivera
}

See end of article for authors' affiliations

\section{Correspondence to:}

Dr E Pascual, Catedrático de Medicina (Reumatología), Hospital General

Universitario de Alicante,

Maestro Alonso 109, 03010

Alicante, Spain;

pascual_eli@gra.es

Accepted 21 December 2006

Published Online First

12 January 2007

\begin{abstract}
Objectives: To determine whether hypouricaemic treatment results in the disappearance of urate crystals from gouty joints and to define the time required.

Methods: In 18 patients with monosodium urate (MSU) crystal proven gout, and after the initiation of successful serum uric acid (SUA)-lowering treatment, an arthrocentesis of the asymptomatic signal joint (11 knees, 7 first metatarsophalangeal joints) was performed every 3 months to obtain a synovial fluid (SF) sample. The sample was then analysed for the presence of MSU crystals, and the number of crystals/400x field was noted. SUA levels and the duration of gout were also noted.

Results: MSU crystals disappeared from the SF of all 18 joints after reduction of SUA to normal levels. The time required for disappearance ranged from 3 to 33 months; disappearance time correlated with the duration of gout $\left(r_{s}=0.71 ; p<0.01\right)$. The median number of MSU crystals in the SF samples before uratelowering treatment was $7.5(2.5-11)$ crystals $/ 400 \times$ field, reducing to $3(1-6.5)$ crystals $/ 400 \times$ field $(p<0.05)$ at 3 months. Crystal counts continued to decrease after 3 months.

Conclusions: In gout, reduction of SUA to normal levels results in disappearance of urate crystals from SF, requiring a longer time in those patients with gout of longer duration. This indicates that urate crystal deposition in joints is reversible. Normalisation of SUA levels results in a decrease in the concentration of MSU crystals in SF in the asymptomatic gouty joints. This may partially explain the reduced frequency of gouty attacks when a patient has been treated with SUA-lowering drugs.
\end{abstract}

G out is a monosodium urate (MSU) crystal deposition disease with strong preference for joints, where the presence of crystals results in episodes of inflammation. While serum uric acid (SUA) levels remain high, gouty attacks become more frequent and affect new joints, and tophi may form and grow. ${ }^{1}$ But, as already noted, ${ }^{2}$ when SUA levels are reduced below normal, tophi size is reduced, the speed of this reduction depending on the SUA levels achieved. ${ }^{3}$ MSU crystals are found in the synovial fluid (SF) of previously inflamed, currently asymptomatic joints of patients untreated with SUAlowering agents ${ }^{5}$; but when SUA levels are reduced, crystals may disappear from the joints. ${ }^{5}$ SUA levels lower than $360 \mu \mathrm{mol} / \mathrm{l}$ are sufficient to promote the dissolution of the crystals. ${ }^{6}$

In this report we show that reduction of SUA levels results in crystal disappearance from the joints in all patients tested, and have determined the time needed for it. Additionally, we gained insight into the effect of reducing SUA levels on the concentration of MSU crystals in the SF samples drawn from these asymptomatic joints.

\section{PATIENTS AND METHODS}

Patients with MSU crystal proven gout and previously untreated with urate-lowering drugs for any significant length of time (occasional short treatment periods, generally received after gouty attacks were allowed) were asked to participate. This request was made after performing an initial arthrocentesis at either a knee or first metatarsophalangeal (MTP) joint, at which time the diagnosis was confirmed, so that patients were aware of the discomfort associated with repeated arthrocenteses required by the study. Arthrocentesis of the asymptomatic knee or first MTP joint has been a routine diagnostic procedure at our clinics, and formed part of the method in our previous studies ${ }^{78}$; in most cases patients had only modest discomfort. Patients diagnosed at the time of an attack, entered the study 2 months after the attack had ended, so all patients were asymptomatic at the study start. In all patients we recorded the time since the appearance of gout in any joint. Patients with tophaceous gout were excluded; none had joint alterations on physical examination attributable to gout, and joint $x$ ray examinations were not carried out. The study was approved by the ethical committee of our hospital and informed consent was obtained from each patient.

At the initial visit, we obtained the following: a synovial fluid sample from the signal joint for analysis, and a blood sample for determination of SUA, a standard complete blood count, and standard laboratory test results, including those assessing the liver and kidney, and a basic urine analysis.

At the initial visit urate-lowering treatment ( 15 benzbromarone, 3 combined benzbromarone and allopurinol) was started with the aim of reducing SUA levels to medium or low normal values. Prophylactic colchicine $1 \mathrm{mg} /$ day was also started at this time. Benzbromarone is a normal treatment for gout at our clinic, and at the time that the study was carried out benzbromarone was still freely available in Spain (at present it has been withdrawn from general use, though in Spain and other European countries remains available for restricted use). None of the patients had had urinary lithiasis. After the first visit patients were reviewed every 3 months. At each visit arthrocentesis of the signal joint was carried out to obtain an SF sample for analysis, and to determine SUA levels; liver and renal biochemistry tests carried out for drug monitoring showed no abnormalities and values are not reported in the

Abbreviations: MTP, metatarsophalangeal; MSU, monosodium urate; SF, synovial fluid; SUA, serum uric acid 


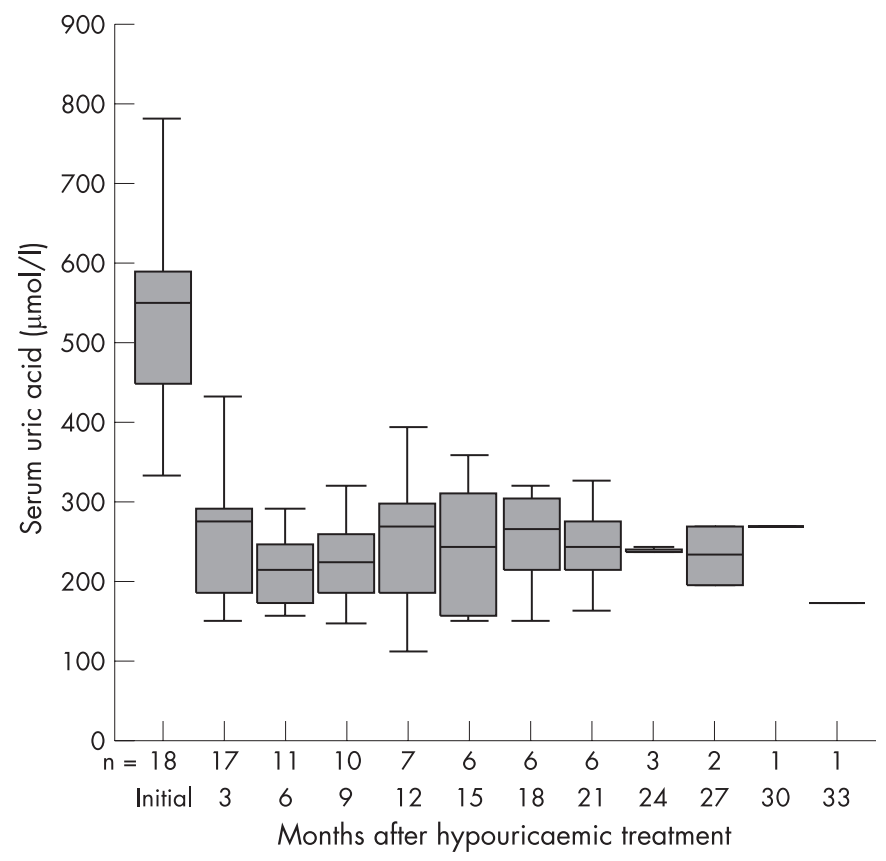

Figure 1 Box plot showing serum uric acid levels at the different visits. The number of patients fell as crystals disappeared from the synovial fluid. Median, 25th to 75th centiles are shown.

"Results" section. Visits continued every 3 months until the SF sample was found to be free of crystals.

For each SF sample a semiquantitative crystal count was carried out, as already reported. ${ }^{5}$ Definitive identification of crystals was made under compensated polarised light. Then, under uncompensated polarised light (where MSU crystals shine brightly and are easily seen ${ }^{9}{ }^{10}$ ) we counted the number of crystals $/ 400 \times$ microscopic field until 10 crystals per field. For statistical calculations we conservatively considered that SF samples with $>10$ crystals per field had 11 crystals. If no crystals were seen in the first field, we searched additional fields until a crystal was found, or a total of 30 fields had been searched without discovering crystals. Routinely, after 30 fields we continued to observe at least another additional 30 microscopic fields; in this extended observation we found a crystal on only one occasion, and we considered the SF sample to be positive for MSU at field 30. For calculations we considered that if crystals were found in the second field, the sample had 0.5 crystals per $400 \times$ field, if in the third field, 0.33 crystals per field, and so on until 0.03 crystals per field if in the 30th field.

\section{Statistical methods}

The Spearman correlation coefficient for non-parametric data, and the Wilcoxon test for paired samples have been used for analysis of the data. To represent SUA levels and number of crystals per $400 \times$ microscope field, median values and 25 th and 75 th centiles are shown.

\section{RESULTS}

Twenty-six patients joined the trial, though eight withdrew before the SF samples obtained every 3 months were free of crystals. Of these eight patients, one started treatment with anticoagulant drugs, one was diagnosed with an advanced malignancy, two were not compliant with their drug, the other four decided to discontinue and were withdrawn from the study at the first or second follow-up visit. The remaining 18 patients who continued with the study-all men (mean (SD) age 55.6 (11.7) years (range 37-78), with disease duration of
2 months to 40 years, mean 10 years (95\% CI 4.8 to 15.5), median 7 years-had arthrocentesis every 3 months, which was continued until the MSU crystals disappeared from the SF sample obtained (11 knees, seven first MTP joints).

SUA levels at entry were a median of 550 (430-630) $\mu \mathrm{mol} / \mathrm{l}$ and at 3 months had reduced to 290 (160-320) $\mu \mathrm{mol} / \mathrm{l}$ $(\mathrm{p}<0.000)$, remaining stable throughout the study (fig 1$)$.

MSU crystals disappeared from the synovial fluid of all 18 patients in whom arthrocentesis was carried out every 3 months after starting SUA-lowering treatment. The time required for crystal disappearance ranged from 3 to 33 months, and showed a good correlation with the time since the initial episode of gout $\left(r_{s}=0.71 ; \mathrm{p}<0.01\right)$. Of interest, nine out of 10 joints of the patients with gout of $<10$ years' duration were free of crystals after 1 year of SUA-lowering treatment and the remaining joint by 18 months.

Crystal counts of all 18 SF samples before starting uratelowering treatment had a median of $7.5(2.5-11)$ crystals $/ 400 \times$ field. At 3 months counts had reduced to a median of 3 (1-6.5) crystals $/ 400 \times$ field $(p<0.05)$. Crystal counts continued to decrease afterwards (fig 2) and, finally, all crystals disappeared from the SF sample.

\section{DISCUSSION}

After urate-lowering treatment, MSU crystals disappeared from the SF of the knee or first MTP joints of all 18 gouty patients in whom arthrocentesis was carried out every 3 months. The time required for crystal disappearance correlated with the duration of the disease, probably because in joints affected for a longer period of time the amount of crystals accumulated is greater and they take longer to dissolve. A trend towards a decreasing SF concentration of crystals in successive 3-month samples preceded their disappearance. Of interest, in patients whose gout started recently, crystal disappearance was reasonably fast. Absence of MSU crystals from the final SF sample does not exclude the possibility that crystals isolated from the joint cavity may remain at the joint, or in the SF at very low concentration, but the results presented strongly suggest that the presence of MSU crystal deposits in the joints can be reversed.

Our data relate only to the joints sampled, and other crystalcontaining joints of the same patient may require different times to become free of crystals. The search for MSU crystals in successive SF samples was unblinded; however, MSU crystals are clearly distinguished under a simple polarised microscope $^{910}$ and we think that the unblinded observationalthough a limitation of the study-is unlikely to represent a significant source of error.

These data are not surprising, because MSU crystals deposited in joints are likely to disappear as tophi do after successful SUA-lowering treatment. The main aim of SUAlowering treatment in gout is to dissolve the crystals, with our current knowledge in the absence of MSU crystals, gouty inflammation is impossible and the disease can be considered cured, with the limitation that if SUA is allowed to rise again above normal values gouty attacks and tophi reappear, ${ }^{11}$ indicating that new crystals have formed.

The rate of reduction of the size of tophi relates to the level of SUA achieved and this is probably related to the rate of dissolution of the crystals in the joints. Thus, the time required for crystal disappearance is a valid measure of the levels of SUA reached by our patients, and reductions in SUA of different magnitude may result in faster or slower dissolution of crystsals. We used benzbromarone quite extensively in our patients because at the time of the study it was the usual SUA-lowering agent at our unit, and at standard doses was more effective than allopurinol at reducing SUA levels. ${ }^{12}$ 


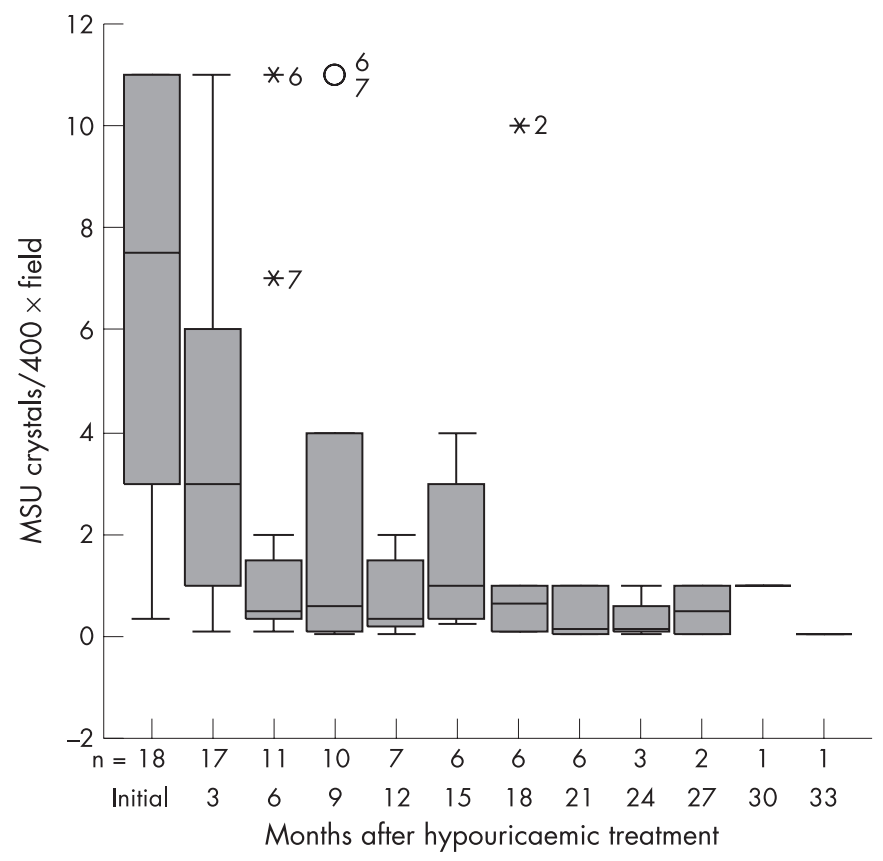

Figure 2 Box plot showing the number of crystals per $400 \times$ microscope field at the different visits. Numbers decrease as crystals disappear from the synovial fluid. Values above 10 crystals per $400 \times$ microscope field are shown as 11 . Median, 25 th to 75 th centiles are shown.

SUA-lowering drugs probably effect crystal dissolution as a result of the low SUA levels; similar results are expected with alternative drugs.

SF samples drawn initially, before starting hypouricaemic treatment, showed a large concentration of crystals in many of the assayed joints. Unfortunately, we had decided to count only up to 10 crystals per microscope field, and seven initial SF samples out of the 18 joints had crystal concentrations clearly above that. Thus, values reported for crystal concentration at the initial samples are artificially low. Despite these shortcomings, initial values are higher than the crystal counts in SF samples drawn at 3 months of SUA-lowering treatment, and numbers decreased further at subsequent visits. This indicates that owing to SUA-lowering treatment, MSU crystals floating in the SF dissolve. Initiation of SUA-lowering drugs (if unaccompanied by prophylactic colchicine or low dose nonsteroidal anti-inflammatory drug treatment) often initially increases the number of gouty attacks, but recurrence of attacks declines afterwards, ${ }^{13}$ even if normal SUA levels are not reached. ${ }^{14}$ The reported decreasing concentration of urate crystals in SF after SUA-lowering treatment may at least be partially responsible for this.

Finally, despite the good understanding that we have of the mechanisms of gout and the reversibility of MSU crystal deposits, standards of gout management on both sides of the Atlantic remain poor. ${ }^{15-17}$ In this report we show that by achieving proper control of serum urate levels, gouty joints can be freed of MSU crystals. This direct evidence may help to stimulate a much needed upgrading of the management of gout by the medical community.

\section{Authors' affiliations}

Eliseo Pascual, Hospital General Universitario de Alicante, and Universidad Miguel Hernández, Alicante, Spain

Francisca Sivera, Hospital General Universitario de Alicante, Spain

Conflict of interest: None declared.

\section{REFERENCES}

1 Yü T-F, Gutman AB. Principles of current management of primary gout. Am J Med Sci 1967;254:893-907.

2 Yï T-F, Gutman AB. Mobilization of gouty tophi by protracted use of uricosuric agents. Am J Med 1951;11:765-9.

3 Perez-Ruiz F, Calabozo M, Pijoan Jl, Herrero-Beites AM, Ruibal A. Effect of urate-lowering therapy on the velocity of size reduction of tophi in chronic gout. Arthritis Rheum 2002;47:356-60.

4 Pascual E. Persistence of monosodium urate crystals, and low grade inflammation, in the synovial fluid of untreated gout. Arthritis Rheum $1991 ; 34: 141-5$.

5 Pascual E, Batlle-Gualda E, Martínez A, Rosas J, Vela P. Synovial fluid analysis for diagnosis of intercritical gout. Ann Intern Med 1999;131:756-9.

6 Li-Yu J, Clayburne G, Sieck M, Beutler A, Rull M, Eisner E, et al. Treatment of chronic gout. Can we determine when urate stores are depleted enough to prevent attacks of gout? J Rheumatol 2001;28:577-80.

7 Pascual E, Castellano JA. Treatment with colchicine decreases the white cell counts in the synovial fluid of asymptomatic knees which contain monosodium urate crystals. J Rheumatol 1992;19:600-3.

8 Pascual E, Jovaní V. A Quantitative study of the phagocytosis of urate crystals in the synovial fluid of asymptomatic joints of patients with gout. $\mathrm{Br} J$ Rheumatol 1995:34:724-6.

9 Lumbreras B, Pascual E, Frasquet J, González-Salinas J, Rodríguez E, Hernández-Aguado I. Analysis for crystals in synovial fluid: training of the analysts results in high consistency. Ann Rheum Dis 2005;64:612-5.

10 Pascual E, Jovani V. Synovial fluid analysis. Best Pract Res Clin Rheumatol 2005; 19:371-86.

11 Loebl WY, Scott JT. Withdrawal of allopurinol in patients with gout. Ann Rheum Dis 1974;33:304-7.

12 Pérez-Ruiz F, Alonso Ruiz A, Calabozo M, Herrero-Beites A, García-Erauskin G, Ruiz-Lucea E. Efficacy of allopurinol and benzbromarone for the control of hyperuricaemia. A pathogenic approach to the treatment of primary chronic gout. Ann Rheum Dis 1998;57:545-9.

13 Shoji A, Yamanaka H, Kamatani N. A retrospective study of the relationship between serum urate level and recurrent attacks of gouty arthritis: evidence for reduction of recurrent gouty arthritis with antihyperuricemic therapy. Arthritis Rheum 2004:51:321-5.

14 Beutler AM, Rull M, Schlesinger N, Baker DG, Hoffman BI, Schumacher HR Jr. Treatment with allopurinol decreases the number of acute gout attacks despite persistently elevated serum uric acid levels. Clin Exp Rheumatol 2001;19:595.

15 Riedel AA, Nelson M, Joseph-Ridge N, Wallace K, MacDonald P, Becker M. Compliance with allopurinol therapy among managed care enrollees with gout: a retrospective analysis of administrative claims. J Rheumatol 2004;31:1575-81.

16 Annemans L, Spaepen E, Nash C, Bonnemaire M, Gilbert T, Malier V. Medical management of chronic gout in the UK and Germany [abstract]. Ann Rheum Dis 2006;65(Suppl 2):430.

17 Pérez-Ruiz F. Treatment of gout before rheumatology consultation is far from that recommended [abstract]. Ann Rheum Dis 2006;65(Suppl 2):436. 